\title{
Case Report of a Clear-Cell Variant of Follicular Thyroid Carcinoma
}

\author{
Yukiomi Kushihashi, 1,2, Kenichiro Ikeda, 1,2,3, Syunya Egawa1,2,3, Yoshiro Saito1,3, \\ Yuya Kurasaa1,3, Takashi Moriya1,3, Sawa Arai'2, Takefumi Yui'2, Hideyuki Katsuta1,2,3, \\ Toshikazu Shimane ${ }^{1,2,3}$ \\ ${ }^{1}$ Head and Neck Oncology Center, Showa University, Tokyo, Japan \\ ${ }^{2}$ Department of Otorhinolaryngology, Showa University School of Medicine, Tokyo, Japan \\ ${ }^{3}$ Department of Oral and Maxillofacial Surgery, Division of Oral Oncology, Showa University, School of Dentistry, Tokyo, Japan \\ Email: ykushihashi32@gmail.com
}

How to cite this paper: Kushihashi, Y., Ikeda, K., Egawa, S., Saito, Y., Kurasaa, Y., Moriya, T., Arai, S., Yui, T., Katsuta, H. and Shimane, T. (2020) Case Report of a Clear-Cell Variant of Follicular Thyroid Carcinoma. International Journal of Otolaryngology and Head \& Neck Surgery, 9, 68-77.

https://doi.org/10.4236/ijohns.2020.92010

Received: January 7, 2020

Accepted: February 24, 2020

Published: February 27, 2020

Copyright $\odot 2020$ by author(s) and Scientific Research Publishing Inc. This work is licensed under the Creative Commons Attribution International License (CC BY 4.0).

http://creativecommons.org/licenses/by/4.0/ (c) (i) Open Access

\begin{abstract}
Clear-cell variants of follicular carcinoma are rare subtypes of thyroid cancer. There is no unified view of the histopathological features of clear cell variants, but follicular carcinomas composed predominantly of clear cells are distinguished from clear cell variants. In clinical practice, it is important to determine whether clear cell variants arise primarily from the thyroid gland or are thyroid metastases of other clear cell carcinomas, such as renal cell carcinoma. We present a case in which a patient with initially suspected anaplastic thyroid carcinoma due to a rapidly progressive anterior neck mass was diagnosed with a clear cell variant of follicular carcinoma after a tissue biopsy. The patient was treated with lenvatinib, then his performance status improved, and he was discharged from the hospital. On day 188 after discharge, a contrast-enhanced computed tomography (CECT) scan of the neck showed further shrinkage of the tumor. However, a CECT scan of the chest revealed multiple lung metastases. On day 233 after discharge, the patient developed severe pneumonia resulting from tracheal rupture due to intratumoral necrosis. It was difficult to decide whether lenvatinib should have been discontinued or reduced when lung metastasis appeared. It is necessary to accumulate additional cases to make informed decisions about continuing lenvatinib therapy.
\end{abstract}

\section{Keywords}

Clear Cell Variant, Follicular Carcinoma, Thyroid Carcinoma, Lenvatinib

\section{Introduction}

Clear cell variants of follicular carcinoma are rare subtypes of thyroid cancer. 
The incidence rate of clear cell variants is about $0.14 \%-3.0 \%$ of thyroid cancer [1] [2] [3]. There is no unified view of the histopathological features of clear cell variants of follicular carcinomas. However, follicular carcinomas composed predominantly of clear cells, a subtype, are distinguished from clear cell variants. In clinical practice, it is important to determine whether clear cell variants of follicular carcinomas arise primarily from the thyroid gland or are a thyroid metastasis of other clear cell carcinomas, such as renal cell carcinoma. Here, we present our experience with a case in which a patient with initially suspected anaplastic thyroid carcinoma due to a rapidly progressive anterior neck mass was diagnosed with a clear cell variant of follicular carcinoma after a tissue biopsy and treated with lenvatinib.

\section{Case}

A 51-year-old man with a history of subacute combined degeneration of the spinal cord, smoking (20 cigarettes per day for 30 years), and drinking (180 - 360 $\mathrm{mL}$ of alcohol daily) had a chief complaint of an anterior neck mass. In X, he was admitted to the neurology department at our hospital for a clinical examination and treatment of gait problems. He was diagnosed with subacute combined degeneration of the spinal cord resulting from vitamin B12 deficiency due to alcohol dependence. An anterior neck mass was seen during the clinical examination. The mass grew rapidly, which gradually reduced his oral intake. He was referred to our department for clinical examination and treatment. At the initial visit, the physical examination revealed a height of $175 \mathrm{~cm}$, a weight of $49 \mathrm{~kg}$, and a body mass index of 16 , showing that he was underweight. Palpation revealed a fist-size mass in the anterior neck region and swelling of multiple lymph nodes on both sides of the neck (Figure 1). Due to the anterior neck mass, mobility related to swallowing movements was impaired, but neither oppressive nor spontaneous pain was detected. His performance status (PS) was 3. Findings from the examination of the larynx and pharynx showed right vocal fold paralysis in the paramedian position without other specific findings.

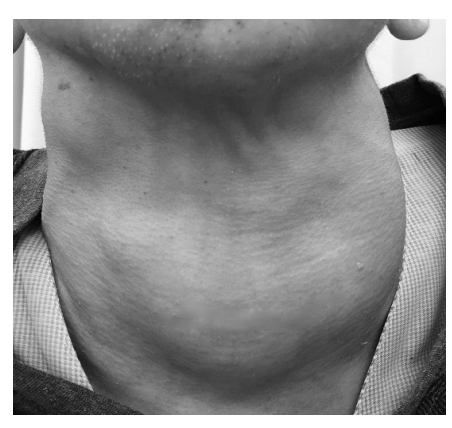

Figure 1. Anterior neck findings at initial consultation. Palpation revealed a fist-size mass in the anterior neck region and swelling of multiple lymph nodes on both sides of the neck. 
Blood biochemical test results were as follows: white blood cells, $5500 / \mu \mathrm{L}$; C-reactive protein, $3.18 \mathrm{mg} / \mathrm{dL}$; hemoglobin, $17.2 \mathrm{~g} / \mathrm{dL}$; platelets, $227,000 / \mathrm{mL}$; albumin, $3.4 \mathrm{~g} / \mathrm{dL}$; calcium, $8.6 \mathrm{mg} / \mathrm{dL}$; lactate dehydrogenase, $543 \mathrm{IU} / \mathrm{L}$; squamous cell carcinoma antigen, $1.5 \mathrm{ng} / \mathrm{dL}$; free triiodothyronine, $2.65 \mathrm{pg} / \mathrm{mL}$; free thyroxine, $0.86 \mathrm{ng} / \mathrm{dL}$; and thyroglobulin, $62,000 \mathrm{ng} / \mathrm{mL}$. Although he had very high levels of thyroglobulin, no abnormality was found in thyroid gland function.

Contrast-enhanced computed tomography (CECT) scans of the neck, chest, and abdomen showed an irregularly shaped, poorly marginated thyroid tumor with an inhomogeneous internal pattern. In addition, there was a lateral displacement of the trachea and esophagus to the left side. Tumor invasion into anterior vertebral muscle and the right side of the tracheal wall was evident, and the tumor surrounded more than half of the outer circumference of the right common carotid artery (Figure 2). There were many lesions that appeared to be metastatic lymph nodes accompanied by peritumoral contrast enhancement on both sides of the neck. Tumor lesions were not found in lung fields or abdominal regions.

The first fine-needle aspiration biopsy produced inadequate specimens, and the second provided specimens that were difficult to use for differential diagnosis.

\section{Course and Treatment}

Clinical course 1:

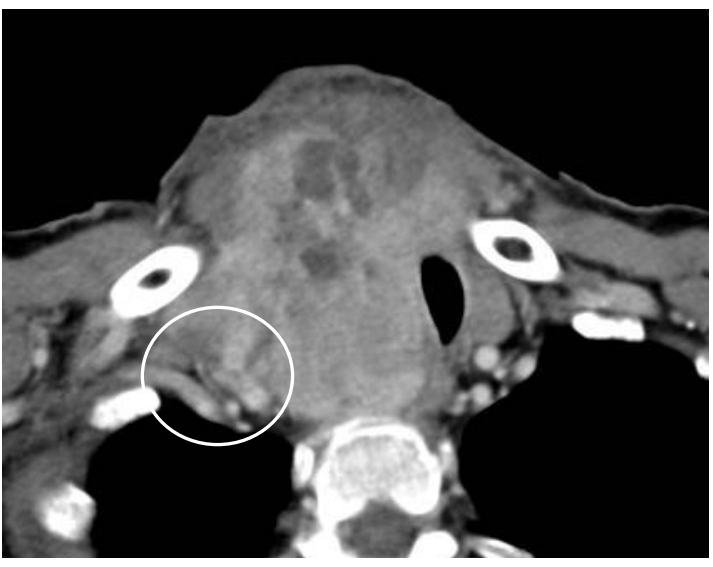

Figure 2. CECT scan of the neck at initial consultation. Contrast-enhanced computed tomography (CECT) scan of the neck at initial consultation. The CECT scan shows an irregularly shaped, poorly marginated tumor with an inhomogeneous internal pattern. In addition, lateral displacement of the trachea and esophagus to the left side is evident. Tumor invasion into anterior vertebral muscle and the right side of the tracheal wall is present, and the tumor surrounds more than half of the outer circumference of the right common carotid artery (०). 
Both fine-needle aspiration biopsies failed to achieve a diagnosis of malignancy. However, we primarily considered the possibility of an undifferentiated rapidly progressive thyroid cancer based on the CECT scan findings of the neck. We explained to the patient and his family members that a tissue biopsy was required for a definitive diagnosis of undifferentiated thyroid cancer, and informed consent was given by the patient. He, however, did not request life-prolonging treatment because he was at risk of sudden deterioration, and the tissue biopsy was performed under a "do not attempt resuscitation" (DNAR) order.

The tissue biopsy was performed under local anesthesia to remove tumor tissue by means of an external incision. The tumor had an abundant blood supply, and bleeding could not be halted easily by cauterization. Tumor tissues were plicated using absorbable sutures, which successfully halted the bleeding.

According to the hematoxylin and eosin staining histopathological findings, numerous clear cells were identified in the follicular structure of the thyroid gland. Epithelial-like tumor cells with relatively large areas of granular eosinophilic cytoplasm possess in oval-shaped nuclei containing prominent nucleoliand irregular-shaped nuclei of different sizes were present. The invasion and proliferation of these tumor cells had a tiny honeycomb-like appearance. Immunostaining was positive for thyroid transcription factor-1 (TTF-1) and negative for thyroglobulin and calcitonin. Ki-67 expression was approximately $10 \%$. Taken together, these findings resulted in diagnosing the patient with a clear cell variant of follicular carcinoma (Figure 3).

Clinical course 2:

Lenvatinib is suggested in the treatment of unresectable differentiated thyroid cancer. After getting a second opinion, the patient canceled the DNA Rorder and gave informed consent for lenvatinib treatment. Because tumor invasion into the common carotid artery and the tracheal wall was present, the tumor board recommended an initial $14 \mathrm{mg}$ daily dose of lenvatinib. On day 8 of hospitalization, thyroglobulin levels increased to $170,000 \mathrm{ng} / \mathrm{mL}$, but later decreased rapidly (Figure 4).

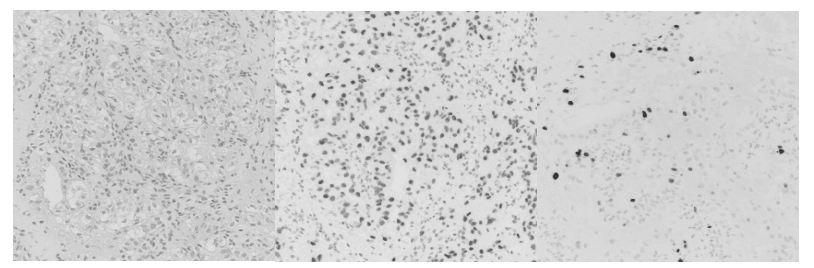

(a)

(b)

(c)

Figure 3. Histopathological findings. (a) H\&E staining; (b) TTF-1; (c) Ki-67. Histopathological findings. (a) Hematoxylin and eosin staining. Numerous clear cells in the follicular structure of the thyroid gland are present. (b) Immunostaining is positive for thyroid transcription factor (TTF)-1. (c) Staining for Ki-67 expression is approximately $10 \%$. 


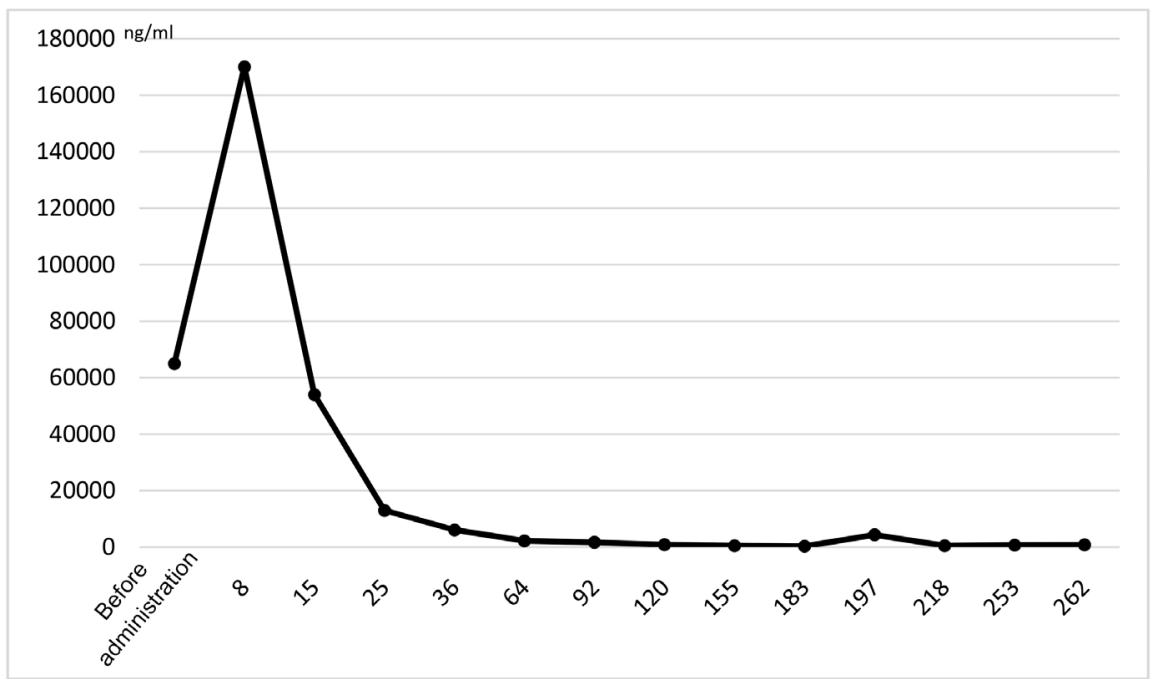

Figure 4. Changes in thyroglobulin levels after administration of lenvatinib. Changes in thyroglobulin levels after administration of lenvatinib. On day 8 of hospitalization, thyroglobulin levels increased to $170,000 \mathrm{ng} / \mathrm{mL}$. Later, thyroglobulin levels decreased rapidly.

Obvious shrinkage of the neck mass was observed on day 28 of hospitalization, and an assessment of the treatment response was conducted using a CECT scan. The results showed poor intratumoral contrast enhancement, tumor diameter shrinkage, expansion of the tracheal lumen, and improvement of tracheal displacement (Figure 5). Adequate oral intake was achieved along with tumor shrinkage, and he improved to PS1. On day 30 of hospitalization, he was discharged from the hospital.

On day 136 after discharge, the anterior neck mass was not clearly detectable by visual inspection (Figure 6) and his thyroglobulin level reached $326 \mathrm{ng} / \mathrm{mL}$. Later, the thyroglobulin levels were maintained around $1000 \mathrm{ng} / \mathrm{mL}$. On day 188 after discharge, a CECT scan of the neck showed further shrinkage of the tumor (Figure 7). However, a CECT scan of the chest revealed multiple lung metastases. Nevertheless, we observed neither worsening of his PS nor any change in respiratory status, and the patient himself did not realize any particular symptoms. Therefore, we decided to continue lenvatinib treatment.

Clinical course 3:

On day 232 after discharge, the patient visited our department as an outpatient presenting with a chief complaint of breathing difficulty and fever the previous day. Urgent computed tomography scans of the neck and chest showed intratumoral necrosis, displacement of the trachea, and pneumonia (Figure 8). We determined that pneumonia was induced by tracheal rupture due to intratumoral necrosis, based on an analysis of the computed tomography image. Under local anesthesia, the anterior neck region was opened immediately, and the pus was drained. Afterwards, multimodal therapy was conducted. At that point, his condition deteriorated rapidly and the patient and his family members choose not to receive life-prolonging treatment. Although multimodal therapy 


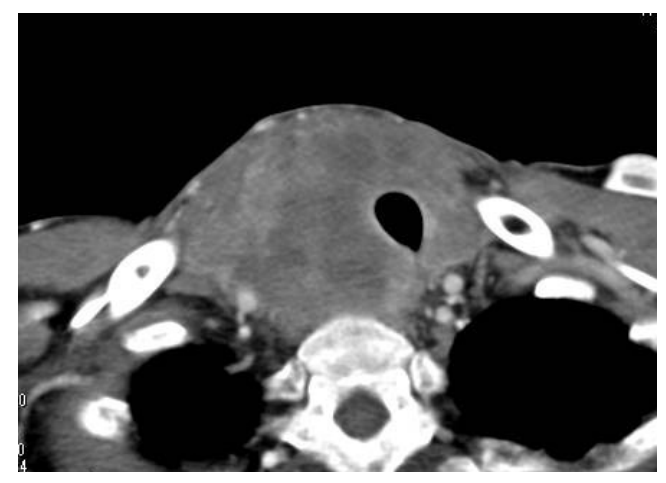

Figure 5. CECT scan of the neck on day 28 of hospitalization. Contrast-enhanced computed tomography (CECT) scan of the neck on day 28 of hospitalization. The CECT scan shows poor intratumoral contrast enhancement, tumor diameter shrinkage, expansion of the tracheal lumen, and improvement of tracheal displacement.

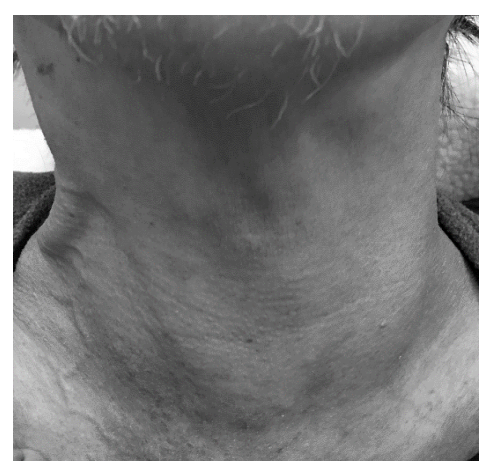

Figure 6. Anterior neck findings on day 136 after discharge (day 166 after initial hospitalization). Anterior neck findings on day 136 after discharge (day 166 after initial hospitalization). The anterior neck mass is not clearly detectable by visual inspection.

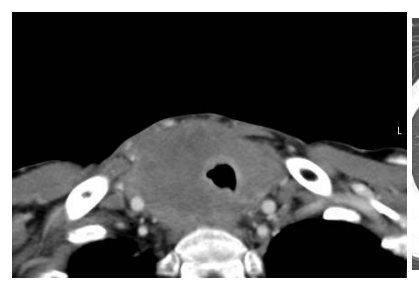

(a)

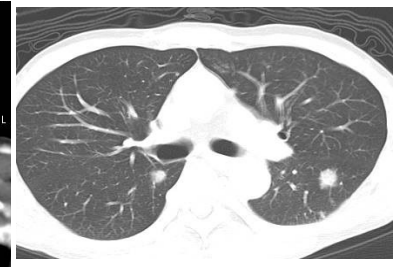

(b)

Figure 7. CECT scan on day 188 after discharge (day 218 after initial hospitalization). (a) CECT scan of the neck; (b) CECT scan of the chest. Contrast-enhanced computed tomography (CECT) scan on day 188 after discharge (day 218 after initial hospitalization). (a) CECT scan of the neck shows further shrinkage of the tumor. (b) CECT scan of the chest shows multiple lung metastases $(\circ)$. 


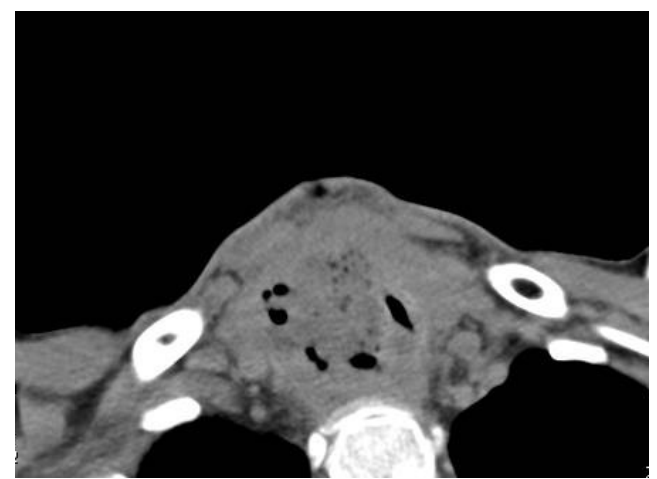

Figure 8. CT scan on day 232 after discharge (day 262 after initial hospitalization). Computed tomography (CT) scan on day 232 after discharge (day 262 after initial hospitalization).

was conducted under a second DNAR order, the patient died after the worsening of his respiratory status on day 233 after discharge.

\section{Discussion}

Clear cell variants of follicular carcinoma are rare types of thyroid cancer; their incidence rate is reportedly $0.14 \%-3.0 \%$ of thyroid cancers [1] [2] [3]. Clear cell variants of follicular carcinoma are classified as special types of follicular carcinoma in the 7th edition of the General Rules for the Description of Thyroid Cancer [4]. In general, many clear cell carcinomas are detected in the kidneys and ovaries. Thus, we must consider the presence or absence of metastases that primarily arise in these organs to distinguish clear cell variants of follicular carcinoma from thyroid metastasis, and a close examination should be performed. Thyroid metastasis from renal cancer is often reported, and its frequency is approximately $5 \%$ of all metastatic foci [5]. In our case, the patient was a male without a history of renal cancer, and a CECT scan did not detect renal tumor lesions. Based on the above-mentioned reasons, along with the pathological findings, the patient was diagnosed with a clear cell variant of follicular carcinoma.

Histopathologically, clear cell variants of follicular carcinoma are diagnosed based on the percentage of predominant clear cells in the carcinoma [6]. Because clear cell follicular carcinoma contains a small number of subcellular organelles, hematoxylin and eosin staining demonstrates many clear hollow spheres. Clear cells contain swollen mitochondria and endoplasmic reticulum. There are only a few reports of immunostaining of clear cell follicular carcinomas, and there are no reports of highly specific stains for this cancer. However, thyroglobulin and TTF-1, highly specific for the thyroid follicular epithelium, are used for staining clear cell follicular carcinomas [7]. In our case, the tumor tissue was positive for TTF-1 and negative for both thyroglobulin and calcitonin.

Surgical resection was initially the first-choice treatment for our patients. However, it was concluded eventually that radical resection was not applicable 
because: 1) the tumor surrounded more than half the circumference of the carotid artery, and 2) tumor invasion into anterior vertebral muscle and the trachea was identified. There are a few reports of prognosis in clear cell variants of follicular carcinoma, but there is no unified view of this prognosis. Clear cell variants of follicular carcinoma seem to have characteristics based mostly on follicular thyroid cancer [8]. Specifically, although there are a few cases of lymphogenous metastasis, there are a relatively large number of cases of hematogenous metastasis. For example, there are many reports that even patients with distant metastasis can live long lives. However, there are also some case reports of unfortunate outcomes caused by rapidly developing disease [7] [8] [9]. In our case, the chief complaint of the patient was a rapidly developing anterior neck mass. Unfortunately, the rapid speed at which the disease progressed resulted in the death of our patient. Furthermore, tumor invasion into surrounding organs, including the trachea, esophagus, carotid artery, tends to cause local recurrence and distant metastasis that are considered markers of a poor prognosis. In the cases of tumor invasion into surrounding organs, tracheal hemorrhage or suffocation can be the leading causes of death, reportedly ranging from $20 \%$ to $30 \%$ [10] [11].

In our case, imaging findings revealed that the patient had a PS of 3 and radically unresectable differentiated thyroid cancer. Therefore, we suggested treatment with lenvatinib, a tyrosine kinase inhibitor covered by Japan's national health insurance for radically unresectable thyroid cancer. To our knowledge, there are no prior reports of lenvatinib treatment for clear cell variants of follicular carcinoma. Because the tumor surrounded more than half the circumference of the right common carotid artery, we explained the risk of fatal hemorrhage associated with tumor shrinkage to the patient and his family members, and received informed consent. A DNAR order was subsequently cancelled, and the treatment commenced. According to a phase III randomized controlled trial (SELECT) of thyroid cancer relevant to lenvatinib therapy, the lenvatinib-treated group had a progression-free survival (PFS) of 14.7 months and showed a 79\% lower risk of disease progression compared to the placebo group [12]. In addition, another report stated that the higher the initial dosage and the longer the treatment duration, the longer the PFS [13]. However, after the Japan national health insurance covered lenvatinib in 2015, this drug was administered to patients with tumor invasion into the common carotid artery and internal jugular vein. Since then, multiple cases of fatal hemorrhage that occurred due to tumor shrinkage and necrosis have been reported [14]. With a focus on PFS, the initial dosage of lenvatinib is $24 \mathrm{mg} /$ day. However, considering the risk of fatal hemorrhage from the carotid artery, the initial dosage in our patient was 14 $\mathrm{mg} /$ day.

According to the SELECT trial, major adverse events of lenvatinib include hypertension (67.8\%), diarrhea (59.4\%), fatigue/weakness (59.4\%), loss of appetite $(50.2 \%)$, loss of body weight (46.4\%), feelings of nausea/sickness (41.0\%), 
and proteinuria $(31.0 \%)$. However, in Japanese patients, the frequencies of hypertension $(86.7 \%)$, proteinuria $(63.3 \%)$, and cutaneous reactions of the extremities (70.0\%) were higher [15]. Our patient had grade 1 hypertension and proteinuria, but no other adverse events were detected. For these reasons, neither cessation nor reduction of lenvatinib associated with adverse events was done.

In our patient, lenvatinib therapy shrank the tumor effectively. Thyroglobulin levels transiently increased from $6200 \mathrm{ng} / \mathrm{mL}$ (before initiation of treatment) to $170,000 \mathrm{ng} / \mathrm{mL}$ (immediately after initiation of treatment), but later decreased rapidly. After reaching the minimum level of $326 \mathrm{ng} / \mathrm{mL}$, thyroglobulin levels were maintained below approximately $1000 \mathrm{ng} / \mathrm{mL}$. The reason for the rapid increase in thyroglobulin levels immediately after the initiation of treatment appeared to be rapid tumor necrosis caused by lenvatinib. Although multiple lung metastases occurred on day 188 after discharge, the patient had a PS of 1 or 2.His condition did not seem to deteriorate and, therefore, the treatment was continued. On day 232 after discharge, the patient experienced breathing difficulty and a computed tomography scan revealed a tracheal rupture due to intratumoral necrosis. At the time of initiating treatment, fatal hemorrhage was a concern due to the observation of tumor invasion into the carotid artery; however, severe pneumonia occurred due to the leakage of intratumoral necrotic tissue from the area of tracheal invasionin to the trachea.

In our case, a DNAR order was made because undifferentiated thyroid cancer was initially suspected, but his PS improved when lenvatinib was introduced. It is controversial whether or not lenvatinib treatment should be discontinued when lung metastases are present. In our case, we continued lenvatinib treatment due to the lack of deterioration of the PS and stabilized progression of the primary tumor. However, eventually, the outcome was unfortunate.

\section{Conclusion}

We report a rare case of a clear cell variant of follicular carcinoma. The patient requested a DNAR order due to rapid disease progression, but his PS improved after the introduction of lenvatinib and he was discharged from the hospital. However, on day 233 after discharge, the patient developed severe pneumonia resulting from tracheal rupture due to intratumoral necrosis, and died. Currently, it is difficult to decide whether lenvatinib should have been discontinued or reduced when lung metastasis appeared. Thus, additional such cases should be accumulated to make informed decisions about continuing lenvatinib therapy.

\section{Conflicts of Interest}

The authors declare no conflicts of interest regarding the publication of this paper.

\section{References}

[1] Carcangiu, M.L., Sibley, R.L. and Rosai, J. (1985) Clear Cell Change in Primary Thyroid Tumors: A Study of 38 Cases. The American Journal of Surgical Pathology, 
9, 705-722. https://doi.org/10.1097/00000478-198510000-00002

[2] Koike, A., Naruse, T., Kanemitsu, T., Kato, K., Kojima, T., Suzumura, K., et al. (1989) Clear Cell Carcinoma of The Thyroid: A Case Report. The Japanese Journal of Surgery, 19, 237-240. https://doi.org/10.1007/BF02471593

[3] Segal, K., Har-El, G., Avidor, I., Shvero, J., Lubin, E. and Sidi, J. (1986) Clear Cell Carcinoma of the Thyroid Gland. Head \& Neck Surgery, 9, 313-319. https://doi.org/10.1002/hed.2890080413

[4] Japanese Society of Thyroid Surgery (2015) General Rules for the Description of Thyroid Cancer. 7th Edition, Kanehara \& Co., Ltd., Tokyo, 18-21.

[5] Saitoh, H. (1981) Distant Metastasis of Renal Adenocarcinoma. Cancer, 48, $1487-1491$.

https://doi.org/10.1002/1097-0142(19810915)48:6<1487::AID-CNCR2820480635>3. $\underline{0 . \mathrm{CO} ; 2-9}$

[6] DeLellis, R.A., Lloyd, R.V., Heitz, P.U., et al. (2004) World Health Organization Classification of Tumors. Pathology and Genetics of Tumors of Endocrine Organs. LARC Press, Lyon.

[7] Motegi, M., Wada, K., Motoyama, C., et al. (2011) A Case Report of Follicular Carcinoma: Clear Cell Variant, Progressing Rapidly to Death. Jibi Inkoka Tembo, 54, 209-215.

[8] Schroder, S. and Bocker, W. (1986) Clear-Cell Carcinomas of Thyroid Gland: A Clinicopathological Study of 13 Cases. Histopathology, 10, 75-89.

https://doi.org/10.1111/j.1365-2559.1986.tb02462.x

[9] Variakojis, D., Getz, M.I., Paloyan, E. and Strauss, F.H. (1975) Papillary Clear Cell Carcinoma of the Thyroid Gland. Human Pathology, 6, 384-390.

https://doi.org/10.1016/S0046-8177(75)80101-8

[10] McConahey, W.M., Hay, I.D., Woolner, L.B., van Heerden, J.A. and Taylor, W.F. (1986) Papillary Thyroid Cancer Treated at Mayo Clinic, 1946 through 1970: Initial Manifestations, Pathologic Findings, Therapy, and Outcome. Mayo Clinic Proceedings, 62, 978-996. https://doi.org/10.1016/S0025-6196(12)62641-X

[11] Kitamura, Y., Shimizu, K., Nagahama, M., Sugino, K., Ozakik, O., Mimura, T., et al. (1999) Immediate Causes of Death in Thyroid Carcinoma: Clinicopathological Analysis of 161 Fatal Cases. The Journal of Clinical Endocrinology and Metabolism, 84, 4043-4049. https://doi.org/10.1210/jcem.84.11.6115

[12] Schlumberger, M., Tahara, M., Wirth, L.J., Robinson, B., Brose, M.S., Elisie, R., et al. (2015) Lenvatinib Versus Placebo in Radioiodine-Refractory Thyroid Cancer. The New England Journal of Medicine, 372, 621-630. https://doi.org/10.1056/NEJMoa1406470

[13] Robinson, B., Schlumberger, M., Wirth, L.J., Dutcus, C.E., Song, J., Taylor, M.H., et al. (2016) Characterization of Tumor Size Changes Over Time from the Phase 3 Study of Lenvatinib in Thyroid Cancer. The Journal of Clinical Endocrinology and Metabolism, 101, 4103-4109. https://doi.org/10.1210/jc.2015-3989

[14] Fukunaga, Y., Kumabe, Y., Nishimura, K., Hatsukawa, H., Kita, S., Morita, T., et al. (2018) A Case of Anaplastic Thyroid Cancer Treated with Lenvatinib and Subsequent Carotid Artery Rupture. Journal of Japan Society for Head and Neck Surgery, 28, 177-182. https://doi.org/10.5106/jjshns.28.177

[15] Kiyota, N., Schlumberger, M., Muro, K., Ando, Y., Takahashi, S., Wirth, L., et al. (2015) Subgroup Analysis of Japanese Patients in a Phase 3 Study of Lenvatinib in Radioiodine-Refractory Differentiated Thyroid Cancer. Cancer Science, 106, 1714-1721. https://doi.org/10.1111/cas. 12826 